\title{
Changing attitudes towards Men's mental health in Merseyside, UK
}

\author{
Walter Brennan* and Edward Cunningham
}

In April 2019, St. Helens was ascribed the title as being the suicide capital in the UK. Figures by the Office for National Statistics [1].

It is also true that men are often three times more likely to commit suicide than women.

In an attempt to understand just why this was the case, we carried out a survey amongst men in the town. In total 117 men were surveyed between January and April 2020.

It found that $44 \%$ (52) of men between the ages of 18 and 55 (117) have or are experiencing mental health issues such as anxiety, depression, or a mixture of both. Just over half of those surveyed (55\%) were unemployed or furloughed due to COVID -19.

Even more worrying is the fact that $71 \%$ (82) of those surveyed struggles to talk, share or even discuss their troubles for the following reasons:

1. Fear of being ridiculed

2. Fear of admitting the problem would make it real

3. Believing that the problem is unique, and others wouldn't understand

4. The myth that being male is about being strong

5. "Not want to burden someone with worry about me"

Anxiety and depression are the two most common mental health issues in the industrialised world today. This paper is about challenging the stigma about mental health that makes sufferers less likely to seek help. According to the World Health Organisation there are almost 800000 people throughout the world who die by suicide every year. Furthermore, for each suicide, there are more than 20 suicide attempts [2].

The impact of COVID-19 may take months if not years for statisticians and social scientists to measure, but at a local level the anecdotal evidence is clear and unless we can change how mental health issues are perceived by men it will only get worse.

In 2016 we developed an innovative programme to introduce the 'mysteries' of interventions such as CBT, Resilience building and Mindfulness to the Cheshire town of Widnes. Working with Widnes Rugby League club we ran a number of drop-in workshops that aimed to smash the stigma of mental ill health, introduce delegates to the major causes, signs, symptoms and long term effects of untreated mental health issues.

The project consisted of three mental health professionals delivering a 2-hour session once per week.
From education - what is depression, anxiety, CBT, ECT!?

To introduction and practicing some of the simple exercises

The interest grew as group sizes increased from 12 to 15 to 19 .

Over the next 8 weeks we introduced a structured programme of learning, laughter, and safety (Table 1).

\section{Findings}

The course was completed by (all 8 sessions) 11 .

Evaluations were:

- Very good $76 \%$

- Good $13 \%$

- Average 3\%

- No good $8 \%$

Anecdotal responses

"Was unsure about the course, but really enjoyed it and would like to learn more about mental health." Steve

"It was really good to do the course within a sports stadium. That made a real difference for me." Andy

"It is not good when there is no carry-on actions. Need a second course I think." Joe

"I found some of the other people proper idiots." Hamed

"I think this should be brought into the workplace" Leo

1. Have you ever felt really nervous and the feelings won't go away? (Figure 1)

(Often -47 Occasionally- 55 Never-13)

2. Have you experienced fast heartbeat, dry mouth, difficulty breathing which you feel is caused by feeling nervous? (Figure 1)

(often -43 occasionally-70 never -3 )

3. Have you ever felt that you are going to faint because of your nervous feelings? (Figure 1)

(often-29 occasionally - 52 never - 34)

*Correspondence to: Walter Brennan, E-mail: oliverbrennan@btinternet.com

Received: September 22, 2020; Accepted: September 29, 2020; Published: October 05, 2020 
4. Have you experienced anxiety at some time in the last 12 months? (Figure 1)

\section{(Yes -72 No 21- Not Sure-18)}

5 . Would you tell a family member if you were feeling really depressed? (Figure 2)

(Yes- 22 No- 82 maybe certain ones- 10)

6. Have you ever felt that suicide is a solution when you've felt depressed? (Figure 2)

(often- 34 occasionally- 41 never-36)

7. Would you go to your GP if you were thinking about hurting yourself? (Figure 2).
(Yes- 40 No - 53 Not sure - 18)

8. If there was a course just for men to talk about depression and anxiety would you attend if it was held (Figure 3 ).

a. At the GP practice -26

b. At the pub- 18

c. At the local sports stadium - 56

d. Somewhere else - 11

Which one would you choose?

${ }^{* *}$ Not all questions were answered

Table 1: Healthy enough to live

\begin{tabular}{|c|c|c|c|}
\hline $\begin{array}{l}\text { Session } 1 \\
\text { Introduction }\end{array}$ & $\begin{array}{l}\text { Course content } \\
\text { Ground rules } \\
\text { Respect }\end{array}$ & Evaluation & $\begin{array}{l}\text { Mind over Mood } \\
\text { Greenberger D Padesky C (1995) } \\
\text { ISBN 0-89862-128-3 }\end{array}$ \\
\hline $\begin{array}{l}\text { Session } 2 \\
\text { What is Anxiety? }\end{array}$ & $\begin{array}{l}\text { Causes } \\
\text { Signs } \\
\text { Symptoms }\end{array}$ & $\begin{array}{l}\text { Discussion } \\
\text { Summary }\end{array}$ & $\begin{array}{l}\text { Cowen P, Harrison P \& Burns T(2012) } \\
\text { SHT of Psychiatry } \\
\text { ISBN 970-0-19-960561-3 }\end{array}$ \\
\hline $\begin{array}{l}\text { Session } 3 \\
\text { What is Depression? }\end{array}$ & $\begin{array}{l}\text { Causes } \\
\text { Signs } \\
\text { Symptoms }\end{array}$ & $\begin{array}{l}\text { Discussion } \\
\text { Summary }\end{array}$ & $\begin{array}{l}\text { Cowen P, Harrison P \& Burns T(2012) SHT } \\
\text { of Psychiatry } \\
\text { ISBN 970-0-19-960561-3 } \\
\text { DSM-5 APA (2013) } 5^{\mathrm{TH}} \text { EDITION } \\
\text { ISBN 978-0-89042-555 }\end{array}$ \\
\hline $\begin{array}{l}\text { Session } 4 \\
\text { How does CBT work? }\end{array}$ & $\begin{array}{l}\text { Myths about thinking } \\
\text { Changing the way we think }\end{array}$ & $\begin{array}{l}\text { Discussion } \\
\text { Summary }\end{array}$ & $\begin{array}{l}\text { Mind over Mood } \\
\text { Greenberger D Padesky C (1995) } \\
\text { ISBN 0-89862-128-3 }\end{array}$ \\
\hline $\begin{array}{l}\text { Session } 5 \\
\text { Examples of CBT and other effective } \\
\text { interventions }\end{array}$ & $\begin{array}{l}\text { Case studies } \\
\text { Exercises } \\
\text { Problem solving }\end{array}$ & $\begin{array}{l}\text { Discussion } \\
\text { Questions Answers } \\
\text { Summary }\end{array}$ & $\begin{array}{l}\text { BUTLER G \& HOPE T (2004) } \\
\text { MANAGE YOUR MIND } \\
\text { ISBN 0-19-852772-1 }\end{array}$ \\
\hline $\begin{array}{l}\text { Session } 6 \\
\text { Resilience } \\
\text { What is it? }\end{array}$ & $\begin{array}{l}\text { Why it matters } \\
\text { How we find it and practice it }\end{array}$ & $\begin{array}{l}\text { Discussion } \\
\text { Questions Answers } \\
\text { Summary }\end{array}$ & $\begin{array}{l}\text { BUTLER G \& HOPE T (2004) } \\
\text { MANAGE YOUR MIND } \\
\text { ISBN 0-19-852772-1 } \\
\text { BRENNAN W(2005) HAVING A } \\
\text { HEALTHY MIND OCCUPATIONAL } \\
\text { HEALTH }\end{array}$ \\
\hline $\begin{array}{l}\text { Session } 7 \\
\text { Problem Solving skills \& Assertiveness }\end{array}$ & $\begin{array}{l}\text { Why these factors are crucial spokes in the } \\
\text { wheel of resilience }\end{array}$ & $\begin{array}{l}\text { Discussion } \\
\text { Questions and answers } \\
\text { Summary }\end{array}$ & $\begin{array}{l}\text { BUTLER G \& HOPE T (2004) } \\
\text { MANAGE YOUR MIND } \\
\text { ISBN 0-19-852772-1 }\end{array}$ \\
\hline $\begin{array}{l}\text { Session } 8 \\
\text { Exercise and mental health }\end{array}$ & $\begin{array}{l}\text { Theory \& Practice } \\
\text { Simple exercises for everyone }\end{array}$ & $\begin{array}{l}\text { Discussion } \\
\text { Questions and answers }\end{array}$ & $\begin{array}{l}\text { LEITH L (1997) } \\
\text { EXERCISING YOUR WAY TO MENTAL } \\
\text { HEALTH, MORGANTOWN, WV:FITNESS } \\
\text { INFORMATION TECHNOLOGY MENTAL } \\
\text { HEALTH }\end{array}$ \\
\hline
\end{tabular}




\begin{tabular}{|l|r|r|r|}
\hline & A & B & C \\
\hline Question1 & 47 & 55 & 13 \\
\hline Question2 & 43 & 70 & 3 \\
\hline Question3 & 29 & 52 & 34 \\
\hline Question4 & 72 & 21 & 18 \\
\hline
\end{tabular}

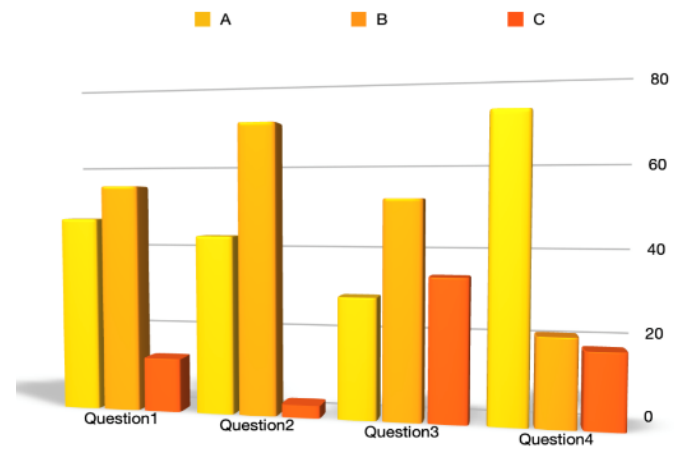

Figure 1. Questions 1-4

\begin{tabular}{|l|r|r|r|r|}
\hline & A & B & \multicolumn{1}{|c|}{ C } \\
\hline Question5 & 47 & 55 & 13 \\
\hline Question6 & 43 & 70 & 3 \\
\hline Question7 & 29 & 52 & 34 \\
\hline
\end{tabular}

$\square \mathrm{A} \quad \square \mathrm{B}$

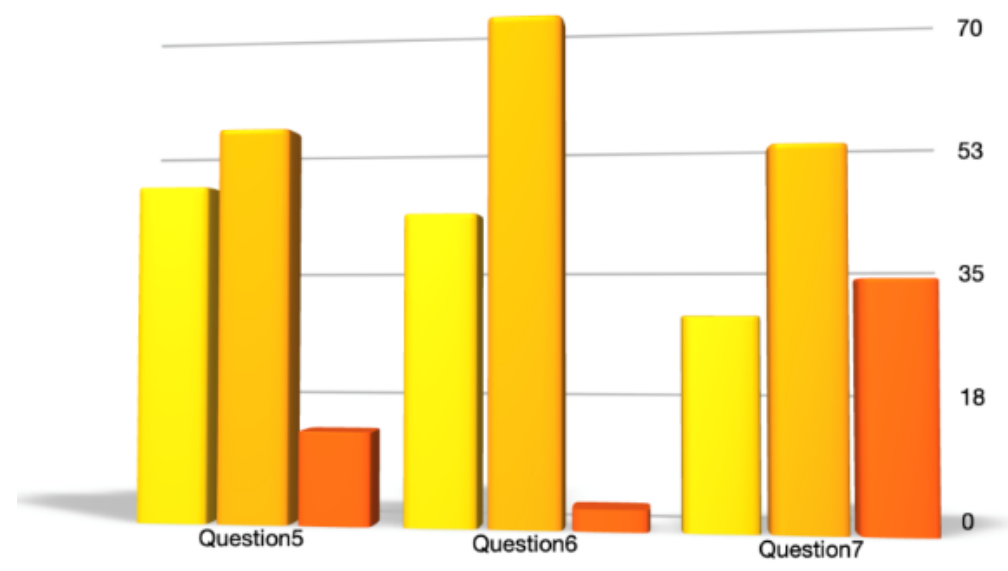

Figure 2: Questions 5-7 


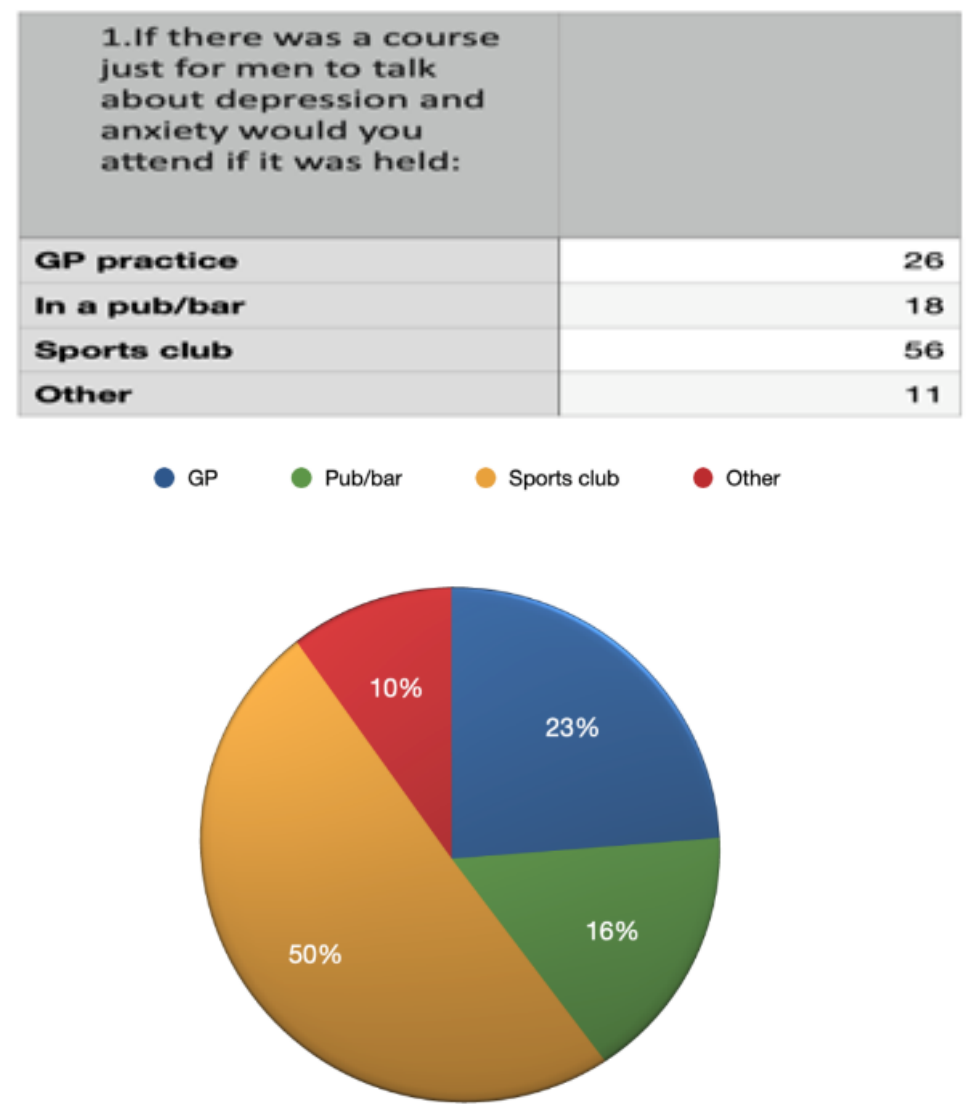

Figure 3. Question 8

\section{References}

1. Suicides in England and Wales: 2019 registrations (2020) Available from: https// www.ons.gov.uk/peoplepopulationandcommunity/birthsdeathsandmarriages/deaths/ bulletins/suicidesintheunitedkingdom/2019registrations

2. Suicide prevention. Available from: https://www.who.int/health-topics/suicide\#tab

Copyright: $\odot 2020$ Brennan W. This is an open-access article distributed under the terms of the Creative Commons Attribution License, which permits unrestricted use, distribution, and reproduction in any medium, provided the original author and source are credited. 